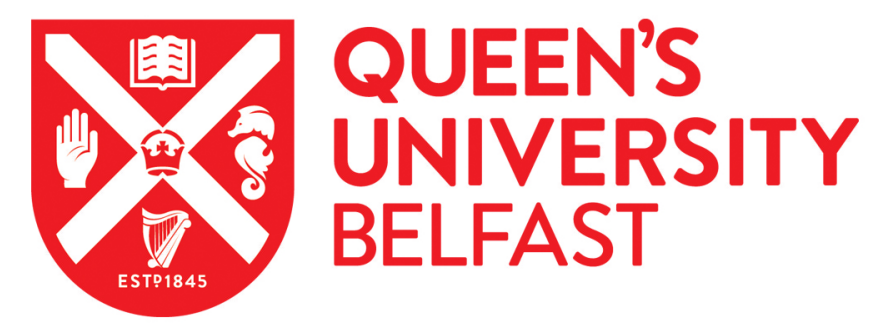

\title{
Determination of the degree of dissociation in an inductively coupled hydrogen plasma using optical emission spectroscopy and laser
} diagnostics

Abdel-Rahman, M., Schulz-von Der Gathen, V., Gans, T., Niemi, K., \& Döbele, H. F. (2006). Determination of the degree of dissociation in an inductively coupled hydrogen plasma using optical emission spectroscopy and laser diagnostics. Plasma Sources Science \& Technology, 15 (4)(4), 620-626. [005].

https://doi.org/10.1088/0963-0252/15/4/005

Published in:

Plasma Sources Science \& Technology

Queen's University Belfast - Research Portal:

Link to publication record in Queen's University Belfast Research Portal

\section{General rights}

Copyright for the publications made accessible via the Queen's University Belfast Research Portal is retained by the author(s) and / or other copyright owners and it is a condition of accessing these publications that users recognise and abide by the legal requirements associated with these rights.

Take down policy

The Research Portal is Queen's institutional repository that provides access to Queen's research output. Every effort has been made to ensure that content in the Research Portal does not infringe any person's rights, or applicable UK laws. If you discover content in the Research Portal that you believe breaches copyright or violates any law, please contact openaccess@qub.ac.uk. 


\title{
Determination of the degree of dissociation in an inductively coupled hydrogen plasma using optical emission spectroscopy and laser diagnostics
}

\author{
M Abdel-Rahman'1, V Schulz-von der Gathen ${ }^{2}$, T Gans ${ }^{2}$, \\ $\mathrm{K} \mathrm{Niemi}^{2}$ and H F Döbele ${ }^{3}$

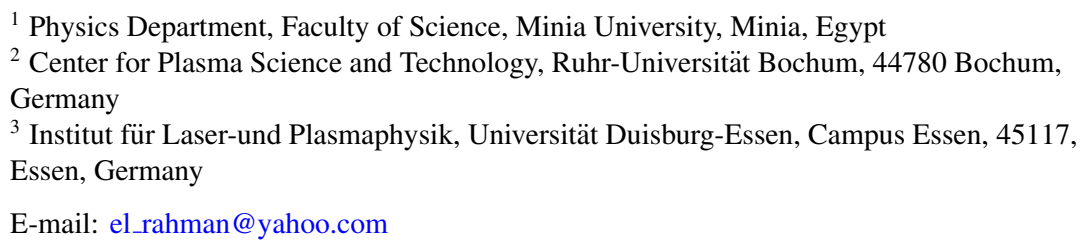

Received 26 April 2006, in final form 14 June 2006

Published 18 July 2006

Online at stacks.iop.org/PSST/15/620

\begin{abstract}
The knowledge of absolute concentrations of radicals in molecular discharges, such as the density of atomic hydrogen in $\mathrm{H}_{2}$ plasmas, is crucial for understanding processes at the surface and is, therefore, of great interest in a variety of plasma applications. Reliable spectroscopic methods are indispensable for the determination of the degree of dissociation in processing plasmas. In this paper, we describe a novel optical emission spectroscopy method called two-gas actinometry (TGA). It is based on using a second actinometer gas to take into account dissociative excitation, which is of particular importance at comparatively low degrees of dissociation. TGA is employed for measuring the characteristic dependence of the degree of dissociation, in a wide range of plasma parameters, in an inductively coupled radio-frequency hydrogen plasma. Absolute densities with high accuracy are obtained using two-photon absorption laser-induced fluorescence spectroscopy.
\end{abstract}

\section{Introduction}

Inductively and capacitively coupled radio-frequency (rf) discharges are the two main types of plasma systems used commercially for surface modification, fabrication of modern integrated circuit devices and thin film deposition [1,2]. Inductively coupled plasmas (ICPs), that can provide high electron density at low neutral pressure, are particularly attractive in the domain of plasma processing [3]. ICPs exhibit a transition between a capacitively coupled mode (E-mode) and an inductively coupled mode (H-mode). Under certain conditions of power and pressure a transition mode (T-mode) is observed. A distinction of the modes is possible from the temporal structure and the intensity of the optical emission [4].
Hydrogen discharges are widely used in many technological plasma processes. They are used in materials treatments such as reduction of oxides on surfaces and deposition of thin films [5].

Optical emission spectroscopy (OES) is one of the most popular plasma diagnostic methods in both research and industry because of its simplicity and accuracy [6]. OES has proved to be a very powerful non-intrusive diagnostic tool used to determine internal plasma parameters such as electron density $n_{\mathrm{e}}$, electron temperature $T_{\mathrm{e}}$ and gas temperature $T_{\mathrm{g}}$. It is also used to infer other more complex plasma parameters such as electron energy distribution functions and degrees of dissociations. Several spectroscopic schemes for corona equilibrium conditions are available to determine the degree of dissociation in hydrogen containing plasmas. The corona 
Determination of the degree of dissociation in an inductively coupled hydrogen plasma

model is based on the assumption that only the plasma electrons have kinetic energies high enough to excite the atoms and molecules, whereas excitation by collisions with other species and cascading processes from higher excited states as well as de-excitation by collisional quenching are neglected, so that the direct electron impact excitation from the ground state is simply balanced by spontaneous emission processes [7, 8]. A major disadvantage of OES is that the analysis relies strongly on accurate atomic and molecular data. This is particularly critical for electron impact excitation cross-sections close to the threshold energy [6]. This energy range, however, is essential in investigations of low-temperature plasmas based on the corona equilibrium.

More direct active laser spectroscopic techniques are based on fewer model assumptions and require less atomic and molecular data. They are, therefore, better suited to accurate quantitative investigations [9]. The major disadvantage compared with OES are relatively high experimental requirements and comparatively long measurement times, making it less favourable for monitoring processing plasmas. Two-photon absorption laser-induced fluorescence (TALIF) is a diagnostic technique that can be used for the detection of light ground-state atoms such as hydrogen, carbon, oxygen, nitrogen and fluorine. Since the first excited levels of these species lie above $6.5 \mathrm{eV}$, a single-photon excitation is only possible with vacuum-ultraviolet (VUV) photons of corresponding wavelengths shorter than $190 \mathrm{~nm} \mathrm{[10].} \mathrm{In}$ practice it is more convenient to apply two-photon absorption with the aid of powerful commercial dye laser systems in combination with frequency doubling and mixing in nonlinear optical crystals. Ground state atoms are excited by simultaneous absorption of two UV laser photons to a higher electronic level according to the two-photon selection rules $[11,12]$.

In the following sections we begin with a description of the experimental configuration of our set-up and diagnostic system. Then we elaborate the assumptions and basic ideas of actinometry and develop modifications by advanced actinometric schemes, taking into account dissociative excitation which is particularly important at comparatively low degrees of dissociation. In the following chapter the characteristic dependence of the degree of dissociation in an inductively coupled rf discharge is measured over a wide parameter range using the improved actinometric schemes. Absolute densities with high accuracy are obtained using TALIF spectroscopy.

\section{Experimental set-up and implemented diagnostics}

\subsection{OES set-up}

The experimental set-up including the arrangement of the optical system for OES is shown in figure 1. The discharge chamber consists of a cylindrical stainless-steel vacuum vessel with four CF-100 ports used for OES and for allowing laser diagnostics. The chamber is evacuated to about $10^{-4} \mathrm{~Pa}$ through a bottom CF-200 port by means of a turbo-molecular pump backed by a two-stage rotary pump. The top port contains a dielectric quartz window separating the plasma and a five-turn antenna coil for inductive coupling. The central

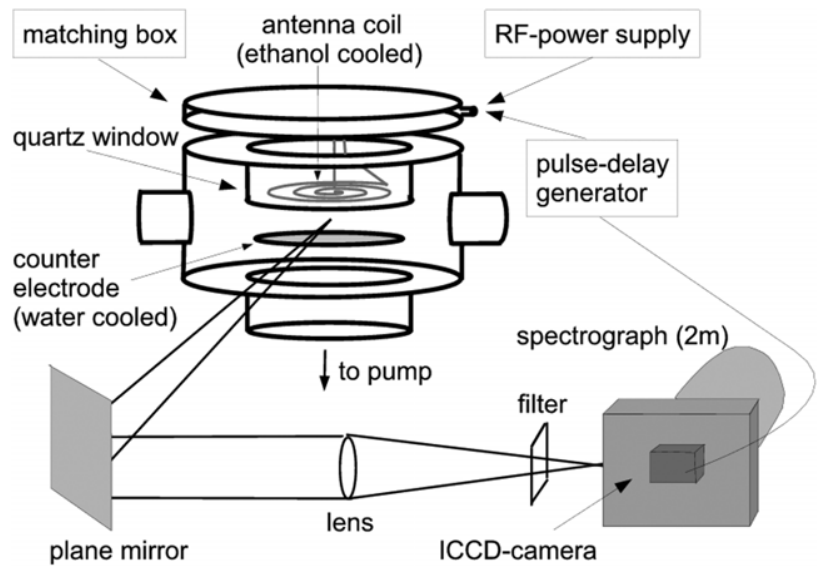

Figure 1. Set-up for OES.

winding of the exciting spiral planar antenna coil is powered at $13.56 \mathrm{MHz}$ using an RF power generator with a matching network, while the outer winding is grounded. The plasma is confined below the quartz window by a grounded electrode. This lower electrode, having a diameter of $100 \mathrm{~mm}$, is made of stainless steel. The optically accessible axial distance between the electrode and the dielectric quartz window is $30 \mathrm{~mm}$. The working gas is $95 \% \mathrm{H}_{2}$ with minority species concentrations of about $3.5 \% \mathrm{Ne}$ and $1.5 \% \mathrm{Kr}$ fed to the discharge through a supplementary port in the lower part of the vessel.

The light emitted from the discharge is imaged with a combination of a plane mirror and one lens $(f=350 \mathrm{~mm})$ onto the entrance slit of a $2 \mathrm{~m}$-spectrograph (Jenoptik PGS 2) as shown in figure 1. The spectrograph is equipped with a 1302 grooves $\mathrm{mm}^{-1}$ grating providing an inverse dispersion of $0.34 \mathrm{~nm} \mathrm{~mm}^{-1}$ at $600 \mathrm{~nm}$. A fast gated intensified charged coupled device (ICCD) camera (LaVision Picostar HR) with an active image array of about $13.2 \mathrm{~mm} \times 8.8 \mathrm{~mm}(576 \times$ 384 pixel) is used as detector. A spectrum of about $4.5 \mathrm{~nm}$ is detected on the CCD chip with a spectral resolution of $0.03 \mathrm{~nm}$. An absolute calibration of the detection system was performed with the aid of a tungsten ribbon lamp (OSRAM WI 17/G).

\subsection{TALIF set-up}

The Spectron SL400 dye laser system ( $\lambda=660-675 \mathrm{~nm})$ is pumped by a frequency-doubled Spectron SL800 Nd:YAG laser $(\lambda=532 \mathrm{~nm})$ as shown in figure 2 . The dye radiation is frequency-doubled in a KDP-crystal and subsequently mixed with the second harmonic of the Nd: YAG radiation in a BBOcrystal to provide UV radiation at $\lambda=205 \mathrm{~nm}$, which is necessary for two-photon excitation of ground state atomic hydrogen. The dye is a mixture of Pyridine 1 and DCM dissolved in propylene carbonate. A maximum UV energy of $160 \mu \mathrm{J}$ within a $10 \mathrm{~ns}$ pulse at a repetition rate of $10 \mathrm{~Hz}$ is obtained. The UV radiation is singled out with a PellinBroca prism and focused with a suprasil lens $(f=30 \mathrm{~cm})$ onto the centre of the discharge vessel. Fluorescence radiation is detected transversal to the laser beam using a Hamamatsu $\mathrm{R} 955$ photomultiplier tube (PMT) with an $\mathrm{H}_{\alpha}(\lambda=656 \mathrm{~nm})$ or krypton $(\lambda=826.3 \mathrm{~nm})$ interference filter in front, respectively. The PMT output is recorded by a fast digitizing oscilloscope (Tektronics TDS 640). The laser energy is monitored with a fast UV diode. 


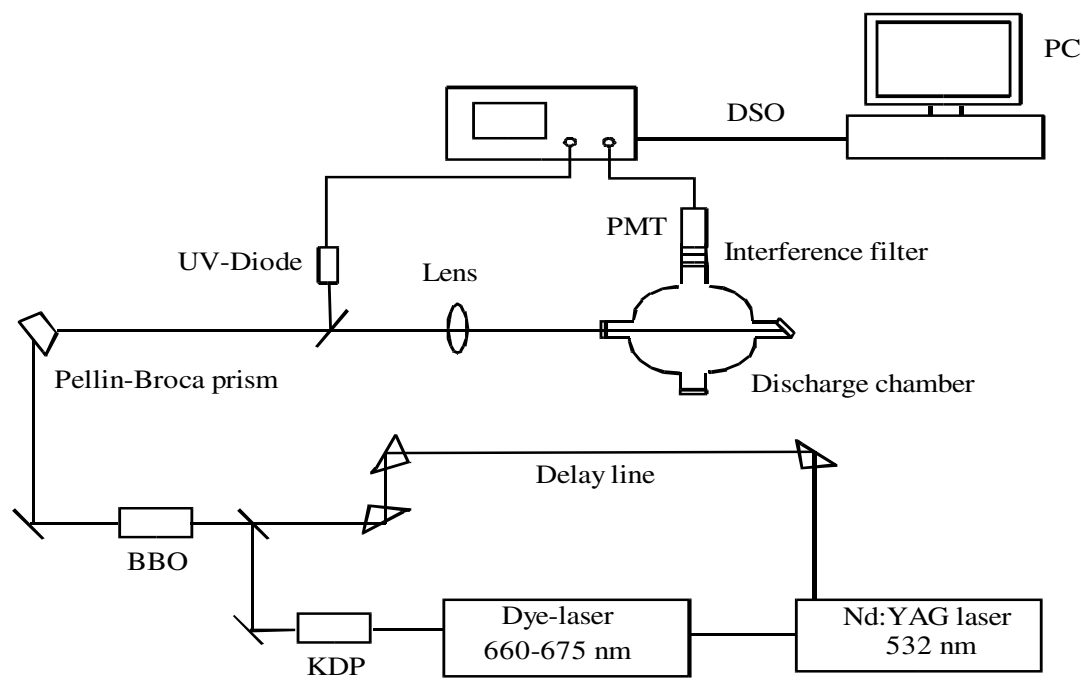

Figure 2. Set-up for two photon laser induced fluorescence.

\section{Actinometry}

\subsection{Classical actinometry}

Actinometry is a method of OES used to measure the density of atoms in their ground state. The method is based on seeding the discharge medium with a small amount of a non-reactive (noble) gas acting as a trace or reference called an actinometer, which has to be properly selected. This technique was firstly introduced by Coburn and Chen in 1980 for an $\mathrm{O}_{2}-\mathrm{CF}_{4}$ plasma [13] in order to measure the density of fluorine atoms using argon as the actinometer.

More general introductions to actinometry and its applicability were published by Gicquel and co-workers [14, 15]. Conditions that must be satisfied in order to obtain reliable measurements are also discussed in detail in these papers and are, therefore, only enumerated here.

1. The emission of both the actinometer and the species of interest are produced by electron impact excitation from their ground state only.

2. The cross-sections of the excitation process for both species have similar energy dependence (shape and threshold).

3. Quenching processes and all other loss processes of the excited states should be negligible compared with spontaneous emission.

4. Only a small amount of the actinometer gas should be introduced to the discharge to avoid perturbations of the plasma.

Under these conditions the influence of the electron energy distribution function $f(E)$ on the excitation function $E_{i}$ (see equation (1)) of a specific state $i$ cancels out considering ratios of the atomic line intensities. The excitation function $E_{i}$ is given by $[6,16]$

$$
E_{i}=n_{\mathrm{e}} \int_{0}^{\infty} \sigma_{i}(E) \sqrt{\frac{2 E}{m_{\mathrm{e}}}} f(E) \mathrm{d} E,
$$

where $n_{\mathrm{e}}$ is the electron density, $m_{\mathrm{e}}$ the electron mass and $\sigma_{i}(E)$ the electron impact excitation cross-section as a function of the electron energy $E$.

Then the atomic density ratio of hydrogen and the actinometer $R$ with the state $j$ can be written using the excitation rates $X_{i}^{H}=n_{0} E_{i}^{H}$ and $X_{j}^{R}=n_{0} E_{j}^{R}$ :

$$
\frac{n_{0}^{H}}{n_{0}^{R}}=\left(\frac{\sigma_{j}^{R} X_{i}^{H}}{\sigma_{i}^{H} X_{j}^{R}}\right)
$$

In the corona equilibrium, the excitation rate $X_{i}$ from the ground state is connected to the measured intensity $I_{\text {meas }}$ of a spectral line emitted from the state $i$ by

$$
X_{i} \alpha \frac{A_{i}}{A_{i k}} N_{\mathrm{cal}, i} I_{\text {meas }},
$$

where $A_{i k} / A_{i}$ is the branching ratio of the specific line and $N_{\text {cal }, i}$ is the wavelength dependent calibration factor of the optical system.

Since the constants of proportionality in equation (3) are the solid angle and the plasma length being equal for all measured lines, we can easily determine the density of the atomic species, in our case hydrogen, from equation (2) as

$$
n_{0}^{H}=n_{0}^{R}\left(\frac{\sigma_{j}^{R} A_{i}^{H} A_{j k}^{R} N_{\mathrm{cal}, i}^{H} I_{\text {meas }}^{H}}{\sigma_{i}^{H} A_{i k}^{H} A_{j}^{R} N_{\mathrm{cal}, j}^{R} I_{\text {meas }}^{R}}\right) .
$$

Here, again, the appropriate ratios, calibration factors and intensities are indicated by the superscripts $H$ and $R$.

The degree of dissociation $\left(K=n_{0}^{H} /\left(n_{0}^{H}+2 \mathrm{n}_{0}^{H_{2}}\right)\right)$ is calculated from the ground state densities of the atomic and molecular hydrogen. However, this classical actinometric method neglects the so-called dissociative channel in which an excited atom in state $i$ is directly generated from the dissociation of the molecule, as discussed in the following section.

\subsection{Actinometry with consideration of dissociative excitation}

In low-temperature hydrogen plasmas the main neutral constituent is $\mathrm{H}_{2}$. Therefore, the dissociative excitation 
process must also be considered since it often yields a very important contribution [17]. Taking into account the dissociative channel, the overall excitation rate $X_{i}^{H}$ of atomic hydrogen can be written as:

$$
X_{i}^{H}=n_{0}^{H} E_{i}^{H}+n_{0}^{H_{2}} E_{i}^{H_{2}} .
$$

Analogous to the derivation of equation (4) we get

$$
n_{0}^{H}=n_{0}^{R}\left(\frac{\sigma_{j}^{R} A_{i}^{H} A_{j k}^{R} N_{\text {cal }, i}^{H} I_{\text {meas }}^{H}}{\sigma_{i}^{H} A_{i k}^{H} A_{j}^{R} N_{\text {cal }, j}^{R} I_{\text {meas }}^{R}}\right)-\frac{E_{i}^{H_{2}}}{E_{i}^{H}} n_{0}^{H_{2}} .
$$

The first term represents the classical actinometry. It does not depend on the electron energy distribution function, if the actinometer is chosen properly, i.e. the ratio of the excitation functions can be replaced by the ratio of the corresponding cross-sections. In contrast, the second term contains the ratio of the excitation functions of both the direct and the dissociative excitation of hydrogen. This term is dependent on the electron energy distribution function because both the direct and dissociative excitation processes generally have cross-sections of different shapes and thresholds. The problem in this second term is connected to the determination of the excitation functions for the direct and the dissociative channel. These excitation functions can be determined in two different ways, either by measuring the EEDF, i.e. with a Langmuir probe, or by applying an improved 'two-gas actinometry' (TGA) method. Knowledge of the EEDF and the cross-sections for both processes allows the calculation of the respective excitation functions and in sequence of the dissociative contribution. The main drawback of this 'probe method' is based on the locality of the probe measurements that have to be incorporated into a line-integrating method such as OES. Therefore, we alternatively introduce an extension of the classical actinometry-the so-called TGA.

\subsection{Advanced two-gas actinometry}

In this advanced actinometry method, we take into account the dissociative excitation of hydrogen by adding a second atomic actinometer. The dissociative excitation function (index $\mathrm{H}_{2}$ ) in the second term can then be compared with the excitation function of the second actinometer state $m$ (index $R 2$ ) assuming the condition of similar cross-sections (threshold and shape) to be fulfilled:

$$
E_{i}^{H}=\frac{\sigma_{i}^{H}}{\sigma_{j}^{R}} E_{j}^{R}
$$

and

$$
E_{i}^{H_{2}}=\frac{\sigma_{i}^{H_{2}}}{\sigma_{m}^{R 2}} E_{m}^{R 2} .
$$

Substituting into equation (6) we get

$$
\begin{aligned}
n_{0}^{H}= & n_{0}^{R}\left(\frac{\sigma_{j}^{R} A_{i}^{H} A_{j k}^{R} N_{\mathrm{cal}, i}^{H} I_{\text {meas }}^{H}}{\sigma_{i}^{H} A_{i k}^{H} A_{j}^{R} N_{\mathrm{cal}, j}^{R} I_{\text {meas }}^{R}}\right) \\
& -n_{0}^{R} \frac{n_{0}^{H_{2}}}{n_{0}^{R 2}}\left(\frac{\sigma_{j}^{R} \sigma_{i}^{H_{2}} A_{m}^{R 2} A_{j k}^{R} N_{c a l, m}^{R 2} I_{\text {meas }}^{R 2}}{\sigma_{i}^{H} \sigma_{m}^{R 2} A_{m k}^{R 2} A_{j}^{R} N_{\text {cal }, j}^{R} I_{\text {meas }}^{R}}\right) .
\end{aligned}
$$

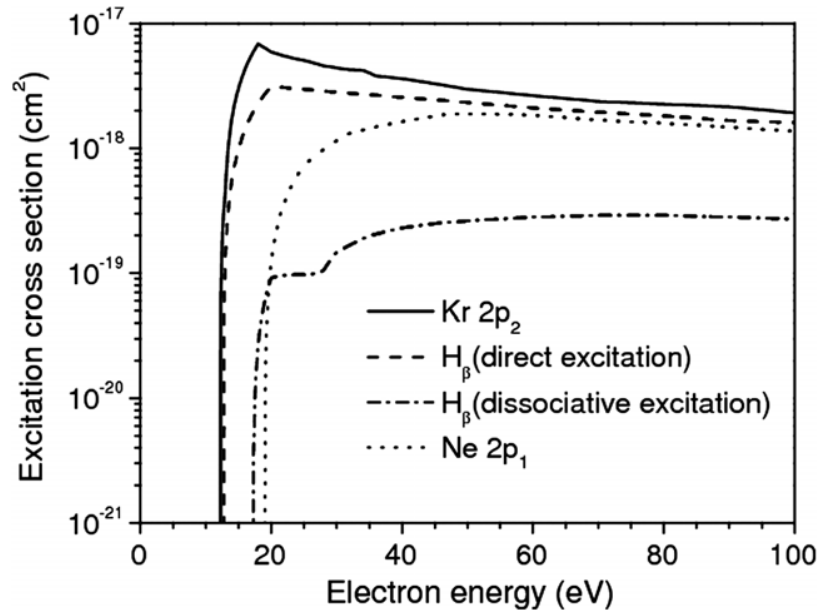

Figure 3. Cross sections of the direct [20] and dissociative excitation [21] of the H-Balmer- $\beta$ emission line and of the corresponding $\mathrm{Kr} 2 \mathrm{p}_{2}$ [22] and $\mathrm{Ne} 2 \mathrm{p}_{1}$ [23] actinometer lines.

Selection of the actinometer lines. For hydrogen discharges the krypton line emitted at $\lambda=826.3 \mathrm{~nm}$ from the $2 \mathrm{p}_{2}$ state $\left(E_{\mathrm{th}}=12.2 \mathrm{eV}\right)$ is considered as a suitable actinometer line for direct excitation of the $n=4$ level of atomic hydrogen that is observed via $H_{\beta}\left(\lambda=486.1 \mathrm{~nm}, E_{\mathrm{th}}=12.7 \mathrm{eV}\right)$. To cover the dissociative excitation of this level $\left(E_{\mathrm{th}}=17.2 \mathrm{eV}\right)$ the emission at $\lambda=585.2 \mathrm{~nm}$ from the neon $2 \mathrm{p}_{1}$ state $\left(E_{\mathrm{th}}=\right.$ $19.0 \mathrm{eV}$ ) was selected. Both pairs show cross-sections with similar thresholds $E_{\mathrm{th}}$ and shapes, respectively, as shown in figure 3. Furthermore, it was taken into account that for these states reliable quenching coefficients, determined by OES and cross checked by laser spectroscopy, are available [18,19]. By substituting $R$ and $R 2$ into equation (7) by $\mathrm{Ne}$ and $\mathrm{Kr}$, the atomic hydrogen density can easily be determined.

\section{Results and discussions}

\subsection{Actinometry results}

The atomic hydrogen density and the degree of dissociation as a function of power at $10 \mathrm{~Pa}$ for the classical, advanced TGA and the 'probe methods' are shown in figures $4(a)$ and $(b)$. The probe measurements of the EEDF at a position about $13 \mathrm{~mm}$ from the grounded electrode are used to calculate the second term of equation (6).

At $10 \mathrm{~Pa}$ and $100 \mathrm{~W}$ the discharge ignites in the low density transition mode (T-mode). For higher powers it switches to the high density inductive mode (H-mode). The probe method yields at a power of $100 \mathrm{~W}$ a non-physical result (negative density), attributed to the fact that the part of the EEDF important for the excitation - the energy range above the threshold-is not reliably measured by the probe. So, if the high energy component of the EEDF, locally measured in the centre of the discharge, is overestimated the contribution of the dissociative channel is overestimated, too. This can finally even result in negative densities, as in the $100 \mathrm{~W}$ case.

At this position within the ICP, the density rises by increasing the applied power. For powers below $250 \mathrm{~W}$ the dependence is nearly linear; beyond this saturation is observed. Maximum density of about $3.5 \times 10^{20} \mathrm{~m}^{-3}$ is measured for 

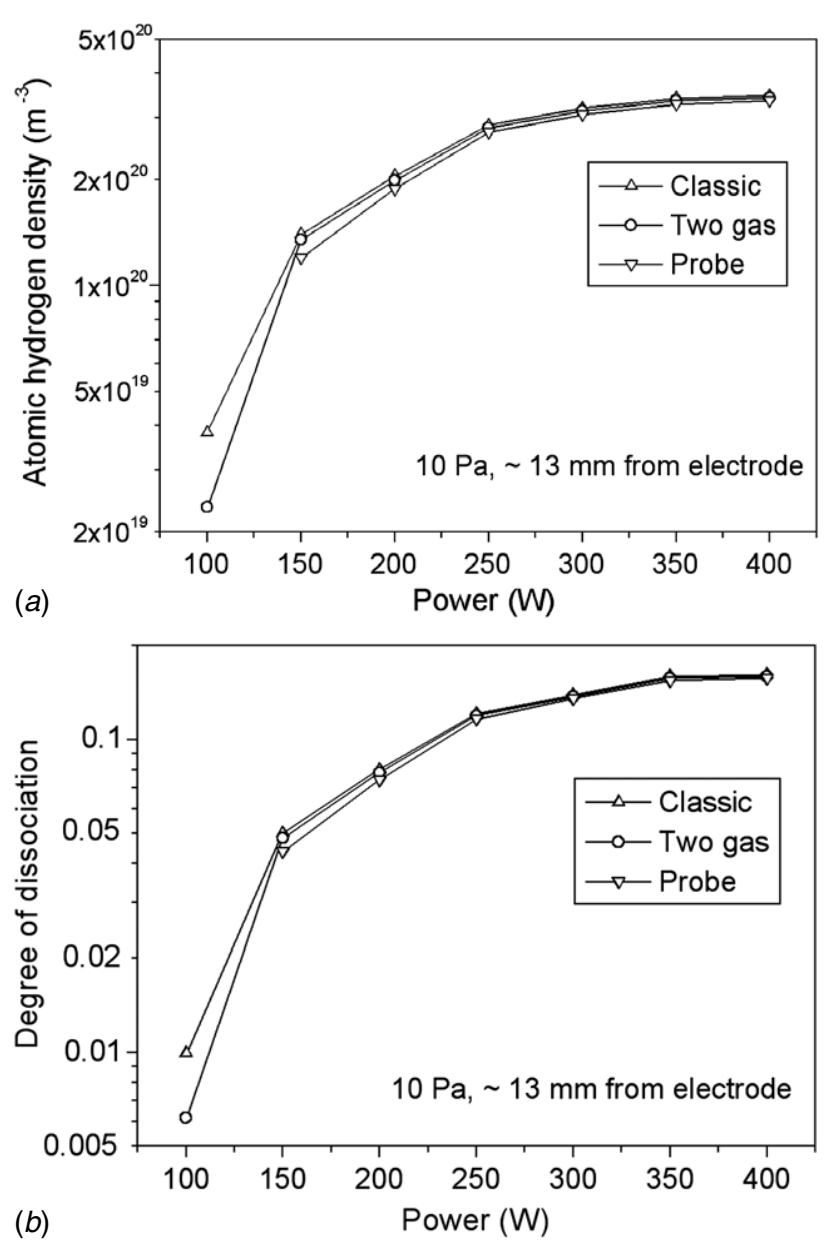

Figure 4. Density and degree of dissociation of different actinometric methods at $10 \mathrm{~Pa}$ as a function of power at the probe position.

$400 \mathrm{~W}$. The classical method generally yields the highest density values as can be expected, since it attributes every signal contribution to the atomic component. The degree of dissociation rises from about $1 \%$ obtained for the classical method at $100 \mathrm{~W}$ to more than $15 \%$ obtained at a power of $350 \mathrm{~W}$. The values of atomic hydrogen density and degree of dissociation obtained by the advanced TGA method are always intermediate between both the results of classical and probe methods. At low powers and atomic densities the deviation between the classical and the other methods is much larger $(\gg 30 \%)$ than for high powers $(<10 \%)$, where the direct contribution becomes dominant as can be easily seen from the absolute values of the cross-sections.

The results of the atomic density and degree of dissociation at $300 \mathrm{~W}$ at different pressures are shown in figures $5(a)$ and $(b)$. In the low pressure range (5-25 Pa), the results of the different actinometric methods coincide. At high pressures (above $25 \mathrm{~Pa}$ ) the density calculated by the probe method deviates significantly from the others. For the density at $60 \mathrm{~Pa}$, the classical method and the advanced TGA method yield a value of about $8 \times 10^{20} \mathrm{~m}^{-3}$, whereas the probe method results in $6 \times 10^{20} \mathrm{~m}^{-3}$. The degree of dissociation shown in figure $5(b)$ reveals a value of about $8 \%$ at $5 \mathrm{~Pa}$ for the classical and the advanced TGA methods. Higher values of about $13 \%$ are obtained within the pressure range from 10
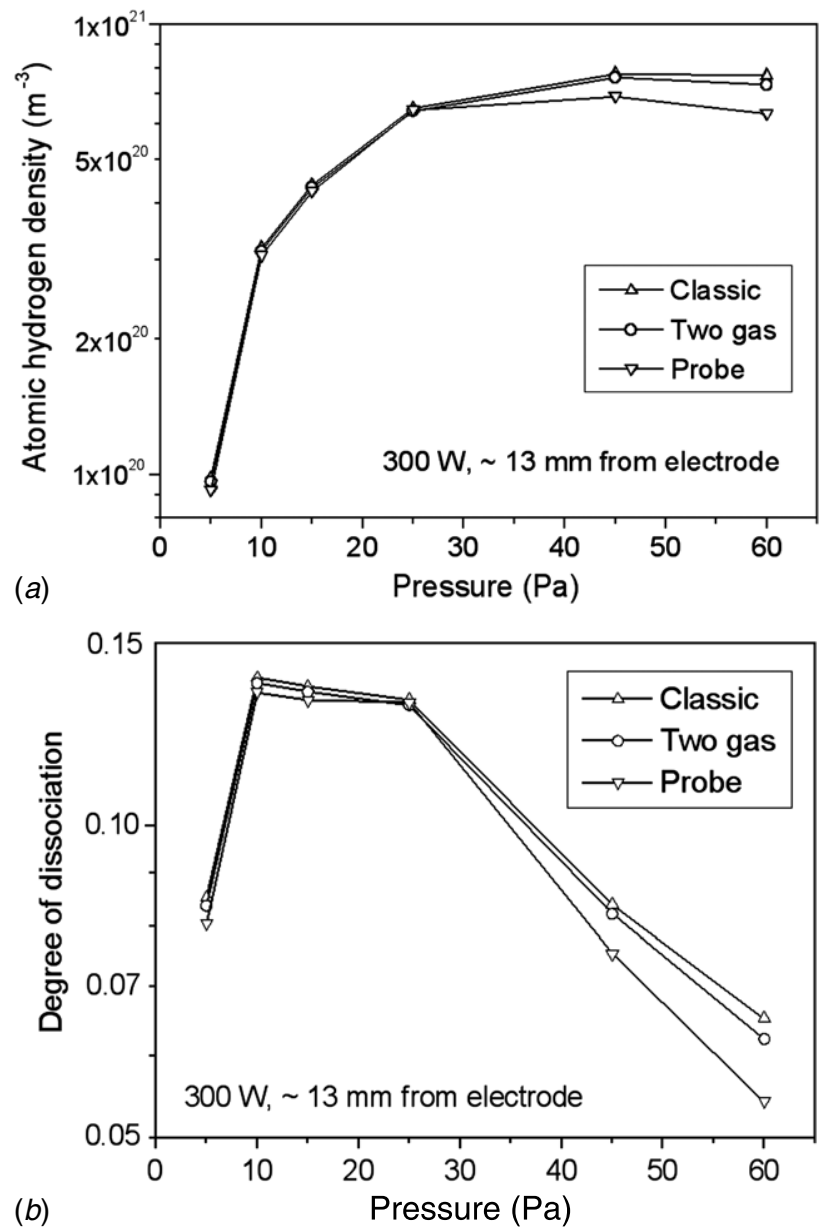

Figure 5. Density and degree of dissociation of different actinometric methods at $300 \mathrm{~W}$ as a function of pressure at the probe position.

to $25 \mathrm{~Pa}$. The degree of dissociation calculated by the three methods decreases rapidly with pressure to its minimum value of about $6 \%$ at $60 \mathrm{~Pa}$. The degree of dissociation obtained by the probe has a slightly lower value, in particular, at higher pressure.

The increasing discrepancy of the probe method results with rising pressure can be attributed to changes in the EEDF. In conclusion, we regard the probe method as questionable, since the probe is an intrusive method, has a limitation in the energy range and yields local and time-integrated results. Therefore, we will not consider the probe results in the following.

Figures $6(a)$ and $(b)$ shows the axial behaviour of the atomic hydrogen density and degree of dissociation in the ICP in the $\mathrm{T}$ - and $\mathrm{H}$-mode. Here, calculations of the density, hence the degree of dissociation, are only available beyond about $8 \mathrm{~mm}$ above the electrode due to intensity limitations caused by the dynamic range of the ICCD camera.

There is no pronounced axial dependence in the atomic hydrogen density either in the T- or H-mode. As can be expected from the foregoing discussion the difference between the densities and hence degrees of dissociation obtained by the classical and the advanced TGA method is more pronounced in the T-mode. The degree of dissociation obtained by the advanced TGA method in the T-mode shows values of about $2 \%$ with deviation of about $20 \%$ less than results obtained by 
Determination of the degree of dissociation in an inductively coupled hydrogen plasma

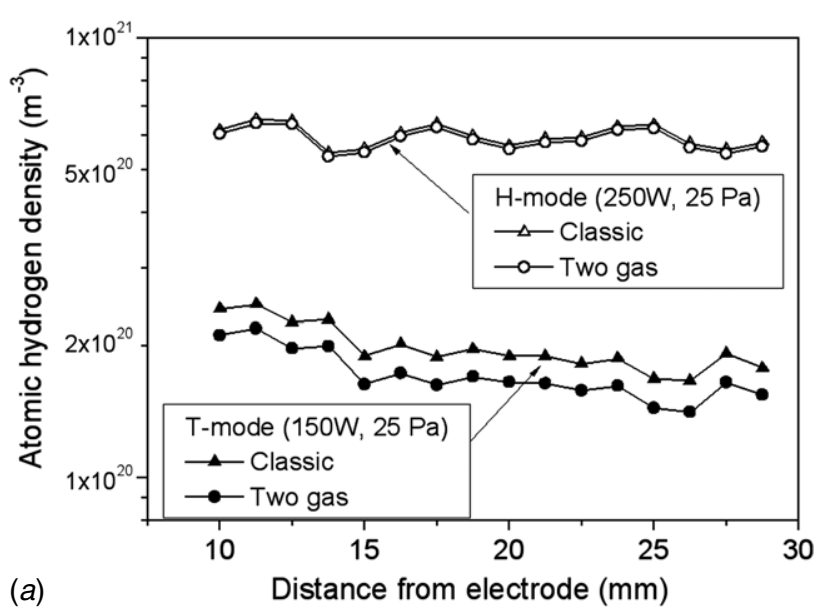

(a)

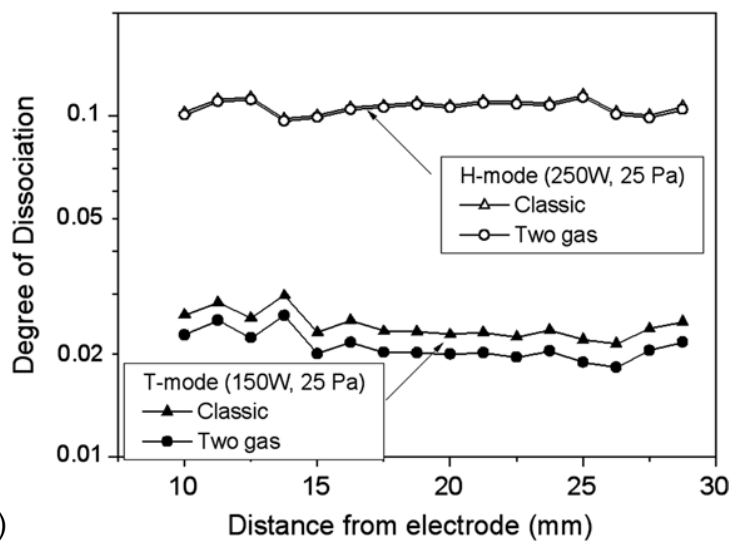

Figure 6. Axial behaviour of (a) the atomic hydrogen density and (b) the degree of dissociation obtained by the classical and advanced actinometry methods in the $\mathrm{T}$ - and $\mathrm{H}$-mode.

the classical method. In H-mode the degree of dissociation has values between 10 and $11 \%$ with a much less difference between the two methods than in the T-mode as shown in figure 6 . The densities coincide (note log. scale) with each other giving an atomic density value of about $6 \times 10^{20} \mathrm{~m}^{-3}$.

It is worth mentioning the different local behaviour of density and degree of dissociation along the axis. This is a result of the temperature profile underlying the calculations. The temperature profile was measured as described in [4] from an analysis of the ro-vibrational structure of the Fulcher bands that are recorded in parallel to the actinometry measurements.

As a conclusion of these actinometry measurements, the advanced TGA method is considered to be a good improvement compared to the other methods, since

- it has less assumptions considering dissociative excitation which is not included in the classical actinometric method,

- it gives line-of-sight integrated values,

- it is a non-intrusive method, i.e. there is no disturbance of the plasma under consideration, and

- it is not restricted to a finite energy range like the probe method but it covers the whole energy range with the given cross-section data.

TGA is, therefore, well suited to monitoring the relative behaviour of atomic densities and the degree of dissociation.

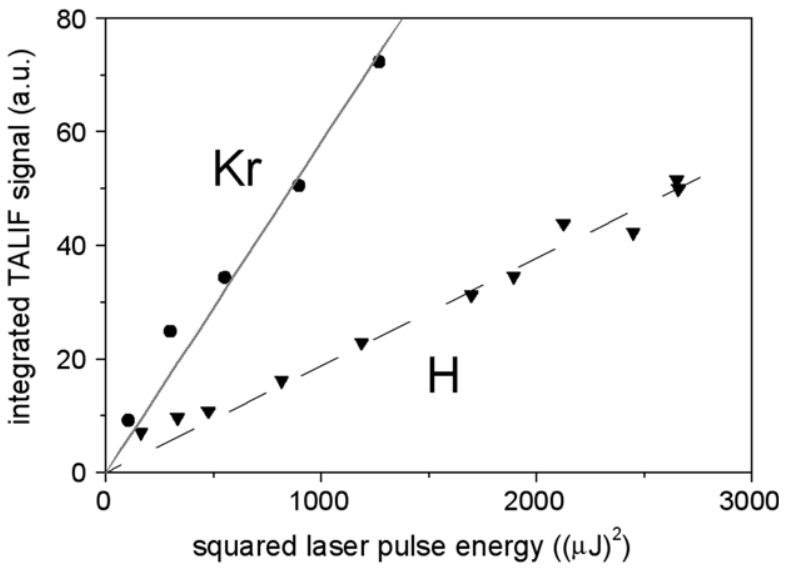

Figure 7. The $\mathrm{H}_{\alpha}$ and Kr TALIF signals as a function of the square of the laser pulse energy.

However, absolute values, as mentioned before, strongly depend on the accuracy of the used data for cross-sections, in particular at energies around the excitation threshold [6,24]. An independent method with high accuracy for absolute values is, therefore, desirable.

\subsection{Comparison with TALIF measurements}

An independent measurement of the absolute atomic hydrogen ground state density by TALIF has been carried out. The wellknown two-photon resonance of atomic hydrogen $\left(1 \mathrm{~s}^{2} \mathrm{~S}_{1 / 2}-3 \mathrm{~d}\right.$ $\left.{ }^{2} \mathrm{D}_{3 / 2,5 / 2}, \lambda=2 \times 205.1 \mathrm{~nm}\right)$ has a corresponding transition in krypton $\left(4 \mathrm{p}^{6}{ }^{1} \mathrm{~S}_{0}-5 \mathrm{p}^{\prime}[3 / 2]_{2}, \lambda=2 \times 204.2 \mathrm{~nm}\right)$ which is used as a stable reference species for the purpose of absolute density calibration [18]. For the $2 p_{2}$ state of krypton $\left(5 p^{\prime}[3 / 2]_{2}\right.$ is the spectroscopic notation. We applied the Paschen notation throughout the text for reasons of simplicity. The fluorescence radiation via the $3 \mathrm{~d}^{2} \mathrm{D}_{3 / 2,5 / 2}-2 \mathrm{p}^{2} \mathrm{P}_{1 / 2,3 / 2}$ hydrogen transition at $\lambda=656 \mathrm{~nm}$ and the $5 \mathrm{p}^{\prime}[3 / 2]_{2}-5 \mathrm{~s}^{\prime}[1 / 2]_{1}$ krypton transition at $\lambda=826.3 \mathrm{~nm}$ is detected.

The $\mathrm{H}_{\alpha}$ TALIF signal from the centre of the discharge as a function of time after switch off the plasma $(250 \mathrm{~W}$ and $25 \mathrm{~Pa}$ ) reveals an exponential decay time of about $3.12 \mathrm{~ms}$ (not shown), which is used to recalculate the density during the stationary on-phase. For calibration a TALIF measurement at a known krypton density (5 $\mathrm{Pa}$ at room temperature, correspondingly $1.2 \times 10^{21} \mathrm{~m}^{-3}$ ) in the vessel (without running the discharge) is performed.

The atomic hydrogen density is calculated from the following equation:

$$
n_{\mathrm{H}}=\gamma\left(\frac{\sigma_{\mathrm{Kr}} a_{i k}^{\mathrm{Kr}} \mathrm{H}_{\text {signal }}}{\sigma_{\mathrm{H}} a_{i k}^{\mathrm{H}} \mathrm{Kr}_{\text {signal }}}\right),
$$

where $\gamma$ is the detection sensitivity, which depends on the quantum efficiency of the photomultiplier tube (PMT, Hamamatsu R995) and the filter transmission for the fluorescence wavelengths:

$$
\gamma=\frac{T_{\mathrm{Kr}} \eta_{\mathrm{Kr}}}{T_{\mathrm{H}} \eta_{\mathrm{H}}}
$$

Here $T_{\mathrm{H}}$ and $T_{\mathrm{Kr}}$ are the transmission of the $\mathrm{H}_{\alpha}$ and $\mathrm{Kr}$ line filters $(50 \%$ and $80 \%$, respectively). The PMT has a quantum efficiency ratio of $\eta_{\mathrm{H}} / \eta_{\mathrm{Kr}}=2.5$ [25]. 
The value of the cross-section ratio $\sigma_{\mathrm{Kr}} / \sigma_{\mathrm{H}}$ is 0.84 and the optical branching ratios of the $\mathrm{H}_{\alpha}$ and $\mathrm{Kr}$ lines are given by $a_{i k}^{\mathrm{H}}=0.88$ and $a_{i k}^{\mathrm{Kr}}=0.95$, respectively, according to [26]. The $\mathrm{H}_{\alpha}$ and $\mathrm{Kr}$ TALIF signals measured $1 \mathrm{~ms}$ after the switchoff of the plasma as a function of squared laser pulse energy are shown in figure 7. From the linear relation of the $\mathrm{H}_{\alpha}$ and $\mathrm{Kr}$ signals we can calculate the slope of these two lines to get the ratio $\mathrm{H}_{\text {signal }} / \mathrm{Kr}_{\text {signal }}=0.32$ and, according to equation (8), the atomic hydrogen density to be about $2.3 \times 10^{20} \mathrm{~m}^{-3}$. The error of this measurement is about $30 \%$. The corresponding degree of dissociation is $4.85 \%$ with a factor of two deviation from the actinometry results. The same factor was obtained in a previous investigation of the degree of dissociation in a capacitively coupled RF hydrogen discharge between OES and TALIF measurements $[25,26]$. Apart from the problems of cross-sections mentioned above, it has also to be taken into account that the TALIF measurements are local in contrast to the line-of-sight OES measurements.

\section{Conclusion}

Densities of atomic hydrogen and the degree of dissociation in an inductively coupled rf hydrogen plasma were measured under parameter variations using optical emission and laser spectroscopy. Advanced TGA, taking into account dissociative excitation, has been developed and compared with classical actinometry and an advanced actinometry, taking into account dissociative excitation using electron 'temperatures' from Langmuir probe measurements. TGA has been found to be the most reliable method over the investigated parameter range. Classical actinometry overestimates the degree of dissociation, in particular at comparatively low degrees of dissociation where the neglected dissociative excitation is particularly pronounced. The advanced actinometry, based on corrections using data from Langmuir probe measurements, tends to underestimate the degree of dissociation. This is mainly due to the uncertainty of the used Langmuir probe data in the energy range close to the excitation threshold and the implementation of local data in the analysis of line integrated OES data. TALIF measurements yield reliable values for absolute densities of atomic hydrogen. A relatively small discrepancy (factor of two) in the TGA measurements can be attributed to uncertainties in the data for the required cross-sections, in particular in the important energy range close to the excitation threshold.

\section{Acknowledgments}

The authors would like to thank C Fischer and J Leistikow for technical support during this work. One of the authors
(MAR) would like to thank the Egyptian mission department for financial support.

\section{References}

[1] Roth J R 1995 Industrial Plasma Engineering (Bristol and Philadelphia: Institute of Physics Publishing)

[2] Hopkins M B and Lawler J F 2000 Plasma Phys. Control. Fusion 42 B189

[3] Lieberman M A and Lichtenberg A J 1994 Principles of Plasma Discharges and Material Processing (New York: Wiley)

[4] Abdel-Rahman M, Gans T, Schulz-von der Gathen V and Döbele H F 2005 Plasma Sources Sci. Technol. $141-10$

[5] Rousseau A, Granier A, Gousset G and Leprince P 1994 J. Phys. D: Appl. Phys. 271412

[6] Schulz-von der Gathen V and Döbele H F 1996 Plasma Chem. Plasma Process. 16461

[7] Behringer K and Fantz U 1999 Contrib. Plasma Phys. 39411

[8] McWhirter R W P 1965 Spectral intensities Plasma Diagnostic Techniques ed R H Huddlestone and S L Leonard (New York London: Academic)

[9] Niemi K, Mosbach T, Döbele H F 2003 Chem. Phys. Lett. 367549

[10] Amorim J, Baravian G and Jolly J 2000 J. Phys. D: Appl. Phys. 33 R51

[11] Döbele H F, Czarnetzki U and Goehlich A 2000 Plasma Sources Sci. Technol. 9477

[12] Döbele H F, Mosbach T, Niemi K and Schulz-von der Gathen V2005 Plasma Sources Sci. Technol. 14 S31

[13] Coburn J W and Chen M 1980 J. Appl. Phys. 513134

[14] Gicquel A, Chenevier M, Hassouni Kh, Tserepi A and Dubus M 1998 J. Appl. Phys. 837504

[15] Thomaz J C, Amorim J and Souza C F 1999 J. Phys. D: Appl. Phys. 323208

[16] Behringer K and Fantz U 1994 J. Phys. D: Appl. Phys. 272128

[17] Lavrov B P, Pipa A V and Röpcke J 2006 Plasma Sources Sci. Technol. 15135

[18] Niemi K, Schulz-von der Gathen V and Döbele H F 2001 J. Phys. D: Appl. Phys. 342330

[19] Gans T, Lin C C, Schulz-von der Gathen V and Döbele H F 2003 Phys. Rev. A 67012707

[20] Walker J D Jr and St John R M 1974 J. Chem. Phys. 612394

[21] Möhlmann G R and DeHeer F J and Los L 1977 Chem. Phys. 25103

[22] Chilton J E, Stewart M D Jr and Lin C C 2000 Phys. Rev. A 6232714

[23] Chilton J E, Stewart M D Jr and Lin C C 2000 Phys. Rev. A 6152708

[24] Fantz U 2002 Contrib. Plasma Phys. 42675

[25] Niemi K 2003 PhD Thesis University of Essen

[26] Gans T 2001 PhD Thesis University of Essen 